INPLASY

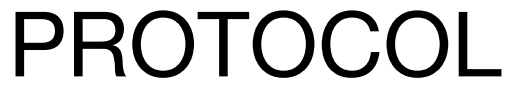

To cite: Holmes et al.

Systematic Review of

Neuropathy and Pelvic

Radiation Disease in Patients

Treated for Cancer. Inplasy

protocol 202070005. doi:

10.37766/inplasy2020.7.0005

Received: 02 July 2020

Published: 02 July 2020

Corresponding author:

Darcy Holmes

darcy_holmes@hotmail.co.uk

Author Affiliation:

University of Nottingham

Support: None.

Review Stage at time of this submission: Preliminary searches.

Conflicts of interest:

No conflicts of interest.

\section{Systematic Review of Neuropathy and Pelvic Radiation Disease in Patients Treated for Cancer}

Holmes, D1; Grainge, M²; West, J3; Morton, A4; Humes, D55.

Review question / Objective: What is the prevalence of neuropathy and pelvic radiation disease in cancer survival patients as a result of chemotherapy and radiotherapy treatment, respectively? PEO framework: P: Cancer survival patients; E: Chemotherapy or radiotherapy; O: Neuropathy or pelvic radiation disease.

Condition being studied: Chemotherapy-induced peripheral neuropathy (CIPN) is paralysis of nerves in the peripheral nervous system and causes loss of motor functions along with numbness and sensitivity to temperature that lead to inhibition of daily tasks and significantly reduced quality of life. Similarly, the manifestations of pelvic radiation disease (PRD) are also debilitating; causing diarrhoea, rectal bleeding, tenesmus, and incontinence. PRD is a collective term that covers radiation-induced conditions in the lower abdomen area such as radiation enteritis, radiation cystitis, and radiation proctitis.

INPLASY registration number: This protocol was registered with the International Platform of Registered Systematic Review and Meta-Analysis Protocols (INPLASY) on 02 July 2020 and was last updated on 02 July 2020 (registration number INPLASY202070005).

\section{INTRODUCTION}

Review question / Objective: What is the prevalence of neuropathy and pelvic radiation disease in cancer survival patients as a result of chemotherapy and radiotherapy treatment, respectively? PEO framework: P: Cancer survival patients; E:
Chemotherapy or radiotherapy; 0 : Neuropathy or pelvic radiation disease.

Rationale: Chance of survival and prolonged survival time for cancers continue to increase, thus the quality of life experienced by survivors must be assessed. Neuropathy and pelvic radiation disease are debilitating diseases and have 
been associated with chemotherapy and radiotherapy, respectively. Therefore, establishing the prevalence of these diseases resulting from cancer treatment can help shape future procedures for cancer treatments and improve the quality of life for cancer survivors.

Condition being studied: Chemotherapyinduced peripheral neuropathy (CIPN) is paralysis of nerves in the peripheral nervous system and causes loss of motor functions along with numbness and sensitivity to temperature that lead to inhibition of daily tasks and significantly reduced quality of life. Similarly, the manifestations of pelvic radiation disease (PRD) are also debilitating; causing diarrhoea, rectal bleeding, tenesmus, and incontinence. PRD is a collective term that covers radiation-induced conditions in the lower abdomen area such as radiation enteritis, radiation cystitis, and radiation proctitis.

\section{METHODS}

Participant or population: Cancer survival patients.

Intervention: Chemotherapy or radiotherapy.

\section{Comparator: n/a.}

Study designs to be included: Primarily observational studies, but no limited to.

Eligibility criteria: Published in 1995 or after. Only adult human cancer studies.

Information sources: Medline and Embase as electronic databases, and Google Scholar for grey literature.

Main outcome(s): Neuropathy and pelvic radiation disease as consequences of chemotherapy and radiotherapy, respectively.

Quality assessment / Risk of bias analysis: The Joanna Briggs Institute (JBI) Critical Appraisal tools.
Strategy of data synthesis: Meta-analysis where studies are homogenous in terms of participants, exposure and outcomes. Descriptive analysis for the studies that are heterogenous and not included in the meta-analysis.

Subgroup analysis: For each outcome, comparisons will be made between male and females, short and long-term consequences, as well as different age groups.

Sensibility analysis: No sensitivity analyses will be conducted.

Language: Limited to English where translations are not available.

Country(ies) involved: United Kingdom.

Keywords: Chemotherapy and Neuropathy. Radiotherapy and pelvic radiation disease.

Contributions of each author:

Author 1 - Darcy Holmes - Drafted the manuscript for the systematic review.

Author 2 - Matthew Grainge - Supervised the project. Read, provided feedback and approved the final manuscript.

Author 3 - Joe West - Second supervisor. Read, provided feedback and approved final manuscript.

Author 4 - Alastair Morton - Screened titles, abstracts, and full text independently from the corresponding author. Contributed to inclusion criteria, data extraction and risk of bias.

Author 5 - David Humes. 